\title{
Diagnosis of pericardial effusion with a new generation hand-carried ultrasound device in cardiothoracic intensive care unit patients
}

\author{
S Schleder ${ }^{1}$, M Dittmar², F Poschenrieder ${ }^{1}$, C Dornia1, C Schmid ${ }^{3}$, J Rennert', \\ C Stroszczynski ${ }^{1}$, EM Jung ${ }^{1}$ and P Heiss ${ }^{1}$ \\ ${ }^{1}$ Department of Radiology; ${ }^{2}$ Department of Anesthesiology; ${ }^{3}$ Department of Cardiothoracic Surgery, University Medical Center \\ Regensburg, Germany \\ Correspondence to: S Schleder. Email: stephan.schleder@ukr.de
}

\begin{abstract}
Background: Technological advances introduced hand-carried ultrasound (HCU) imagers in daily clinical workflow providing several benefits such as fast bedside availability and prompt diagnosis. Purpose: To evaluate the diagnostic yield of a latest generation HCU imager compared to contrast-enhanced multidetector computed tomography (MDCT) for the detection of pericardial effusion (PE) in cardiothoracic intensive care unit (ICU) patients.

Material and Methods: Thirty-six patients from a cardiothoracic ICU were enrolled to this study irrespective of their underlying disease. All patients were examined with a new generation HCU for the presence of PE. Definite diagnosis of PE was based on findings of MDCT as standard of reference. Statistical analysis was performed using PASW 18.

Results: PE was identified in 20 patients by MDCT (prevalence 56\%). The HCU examination was carried out technically successfully in all patients. Sensitivity, specificity, positive and negative predictive value of HCU for the diagnosis of PE were $75 \%, 88 \%, 88 \%$, and $74 \%$, respectively.

Conclusion: HCU provides rapid, practical, reliable, and cost-effective diagnosis of PE in patients on cardiothoracic ICU.
\end{abstract}

Keywords: Ultrasound, thorax, CT, heart, cardiac

Submitted May 17, 2012; accepted for publication August 22, 2012

For patients in the intensive care unit (ICU) pericardial effusion (PE) is a common complication soon after cardiothoracic surgery and its incidence is dependent on the timing of the examination after surgery and is reported to be as high as $85 \%(1,2)$. As an even more severe complication for patients in the cardiothoracic ICU, an early cardiac tamponade is depicted in up to $6 \%$ of patients after cardiac surgery, in most cases owing to postoperative bleeding (3), whereas late cardiac tamponade is less frequent $(4,5)$. Furthermore, PE occurs in up to $21 \%$ of patients suffering from cancer and these patients do also often present with cardiac tamponade (6).

Since 1954, when introduced by Edler and Hertz (7), the diagnosis and evaluation of PE has been based on echocardiography, which is the most effective non-invasive tool for the evaluation of pericardial fluid accumulation (8-10).
However, the bulkiness and heaviness of ultrasound machines limited their bedside use for a long time $(11,12)$ and the first portable ultrasound imagers had limitations in functionality and image quality and even missed important clinical findings (13).

Nowadays, further technological advances generated miniaturized ultrasound systems, leading to the introduction of hand-carried ultrasound (HCU) imagers in daily clinical workflow. Principal advantages of modern HCU devices are fast bedside availability and excellent transportability allowing prompt diagnosis and immediate therapeutic measures combined with low costs (14-17). The disposability of an HCU device and its easy transportability might fasten up the diagnosis of PE or cardiac tamponade at the bedside in the cardiothoracic ICU and help save precious time in coping with urgent situations. 
Table 1 Basic demographic characteristics of enrolled patients

\begin{tabular}{lcc}
\hline & $\boldsymbol{n}$ & $\%$ \\
\hline Patients & 36 & 100 \\
Gender & 21 & \\
Male & 15 & 58 \\
Female & $65(36-87)$ & 42 \\
Median age and age range (years) & $79(58-104)$ & \\
Median weight and weight range (kg) & 3 & 8 \\
Patients without prior surgery/no & & \\
$\quad$ surgery planed & 33 & \\
Patients with prior surgery & $3(1-6)$ & \\
Median time interval and time range & & \\
$\quad$ between surgery and examination (days) & & 36 \\
Type and frequency of surgery & 12 & 24 \\
Coronary bypass & 8 & 15 \\
Cardiac valve prosthesis & 5 & 15 \\
Lung lobectomy & 5 & 6 \\
Pleurodesis & 2 & 3 \\
Ascending aortic replacement & 1 & \\
Thymectomy & & \\
\hline
\end{tabular}

Therefore the aim of the study was to evaluate the diagnostic yield of a latest generation HCU imager compared to contrast-enhanced multidetector computed tomography (MDCT) for the detection of pericardial effusion in cardiothoracic ICU patients.

\section{Material and Methods}

\section{Patients}

From February 2011 to April 2011 a total of 36 consecutive patients admitted to a cardiothoracic surgery ICU for whom a MDCT scan was ordered by their ward physician were examined additionally with HCU for the presence or absence of PE, irrespective of their underlying disease. There was no exclusion criterion. All patients enrolled to the study were examined at the core service hours of the Department of Radiology, i.e. 08:00 to 17:00 on working days and the ultrasound examination was performed by only one examiner (SS). Study participant operators (SS, FP) were neither aware of the indication for the contrast-enhanced MDCT nor of patients' underlying disease. In Table 1 patients' basic demographic data are reported. Informed consent was obtained for all patients. The study was approved by our institution's ethics committee (Ethics Committee of the Medical Faculty, University Medical Center Regensburg, Germany).

\section{Contrast-enhanced multidetector CT scan}

Indications for contrast-enhanced MDCT scan are reported in Table 2. According to standardized examination protocols, all scans were acquired either on a 16-slice CT (SOMATOM Sensation 16, Siemens Healthcare AG, Erlangen, Germany) or on a 128-slice CT (SOMATOM Definition Flash, Siemens Healthcare AG, Erlangen, Germany) after intravenous contrast medium injection of 70-120 mL Accupaque 300 (GE Healthcare, Waukesha, WI, USA) or 60-100 mL Ultravist 370 (Bayer Vital GmbH, Leverkusen, Germany), respectively. MDCT scans were
Table 2 Indications for contrast-enhanced MDCT

\begin{tabular}{lll}
\hline Indication for contrast-enhanced MDCT & $\boldsymbol{n}$ & \% \\
\hline Suspicion of pulmonary embolism & 8 & 22 \\
Suspicion of pneumonia & 8 & 22 \\
Clarification of obscure infection focus & 5 & 14 \\
Suspicion of sternal dehiscence & 5 & 14 \\
Suspicion of mesenteric ischemia & 3 & 8 \\
Suspicion of sternal wound infection & 3 & 8 \\
Suspicion of acute arterial bleeding & 2 & 6 \\
Suspicion of aortic dissection & 2 & 6
\end{tabular}

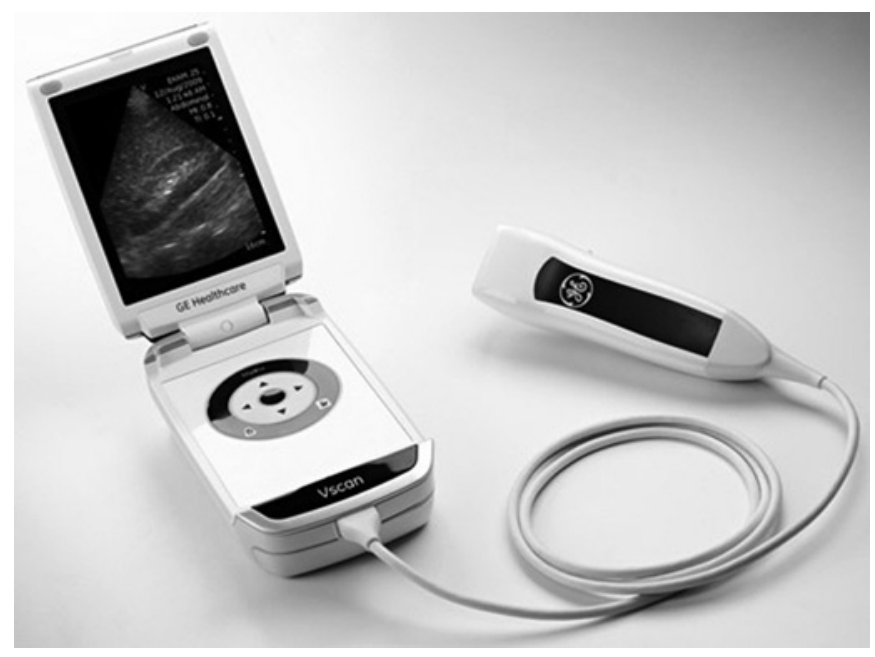

Fig. 1 Image of the study hand-carried ultrasound device (VScan, GE Healthcare, Waukesha, WI, USA) with kind permission of Mr Bastian Werminghoff (GE Healthcare Germany, Munich, Germany)

evaluated for the presence or absence of $\mathrm{PE}$, and the amount of PE was furthermore visually assessed, by the same radiologist $(\mathrm{FP})$, specialized in cardiopulmonary imaging for 4 years who was unaware of the ultrasonographic or clinical findings.

\section{Chest ultrasound with hand-carried ultrasound}

The ultrasound examination was carried out using a modern HCU device (VScan, GE Healthcare, Waukesha, WI, USA) providing a plane $1.7-3.8 \mathrm{MHz}$ transducer for two-dimensional imaging and a 3.5-inch color LCD display. The weight of this imager is $390 \mathrm{~g}$ and the maximum scan time with fully charged batteries is approximately $1 \mathrm{~h}$. A picture of the HCU device is shown in Fig. 1. The chest ultrasononographic studies with the HCU imager were performed just prior to the acquisition of the contrast-enhanced CT scan, when the patient was already lying on the CT table. To standardize and simplify the ultrasonographic examination each patient was examined in supine position when the patient was lying on the CT table. This course of examination was chosen in order to adapt the conditions of examination to the settings in patients on ICU. Assessment of pericardial effusion was made with B-Scan two-dimensional imaging in standard views parasternal long- and short-axis, apical four-chamber view, and subcostal views. PE appears as an anechoic stripe around the heart. Few amounts of PE appear as a thin stripe 

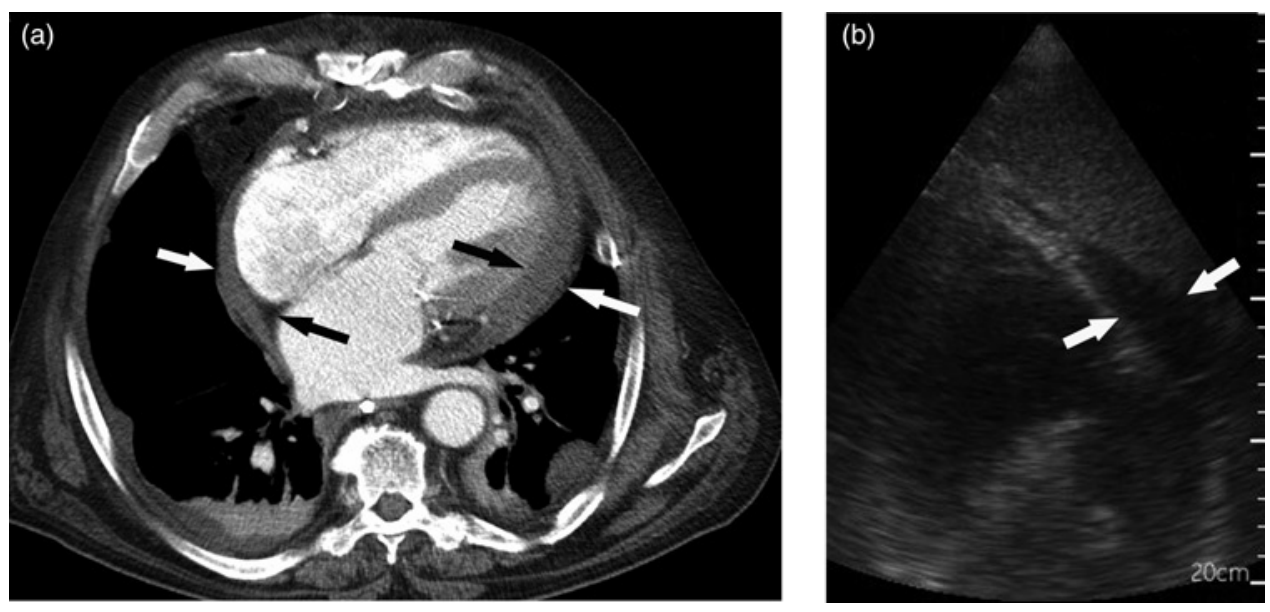

Fig. 2 Congruent finding of a large PE by HCU and contrast-enchanced MDCT scan in a 74-year-old male patient 4 days after cardiac valve prosthesis for whom

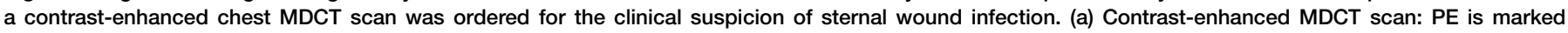
between arrows; (b) B-Scan HCU image (subcostal view): PE is marked between arrows

inside the pericardial space, not extending fully around the heart. Pitfalls in the assessment of PE are that few amounts of PE should not be confused with the pericardial fat pad, and that PE in general may not be mistaken for pleural effusion (18).

The operator (SS) was a radiologist with the knowledge of more than 1000 documented ultrasound examinations within the last year under the supervision of an experienced ultrasound examiner who has conducted $>5000$ ultrasound examinations every year for $>10$ years. Moreover, the operator was unaware of the MDCT scan results or the clinical findings.

\section{Statistical analysis}

Data were acquired using Excel tables (Excel 2007, Microsoft, Redmond, WA, USA). Statistical analysis was performed using PASW 18 (PASW V.18, IBM SPSS Inc., Armonk, NY, USA). Agreement between the findings of $\mathrm{HCU}$ an MDCT as standard of reference was assessed from $2 \times 2$ tables.

\section{Results}

HCU was carried out technically successful in all 36 patients. Mean scanning time with HCU was 1.5 min with a range of 1-3 min.

Contrast-enhanced MDCT scan depicted PE in 20 of 36 patients, hence, prevalence of PE was calculated to be $56 \%$.

Examination with HCU (Fig. 2) revealed PE correctly in 15 of 20 patients $(75 \%)$. In the remaining five cases in whom HCU was false-negative, the amount of PE visually assessed on the MDCT scans revealed $<20 \mathrm{~mL}$ of PE in all five cases. These small amounts of PE were considered to be either physiologically or at least hemodynamically irrelevant.

The absence of PE was correctly verified by HCU in 14 of $16(88 \%)$ patients. HCU was false-positive in two cases in whom the amount of PE was estimated to be $<20 \mathrm{~mL}$, respectively. These small amounts of PE were considered
Table 3 Sensitivity, specificity, positive (PPV) and negative (NPV) predictive value for the diagnosis of pericardial effusion (PE) using hand-carried ultrasound with MDCT as standard of reference

\begin{tabular}{ll}
\hline & $\begin{array}{l}\text { Diagnosis } \\
\text { of PE (\%) }\end{array}$ \\
\hline Sensitivity & 75 \\
Specificity & 88 \\
PPV & 88 \\
NPV & 74 \\
\hline
\end{tabular}

to be physiologically or at least hemodynamically irrelevant as well.

Overall, sensitivity, specificity, positive and negative predictive value of $\mathrm{HCU}$ for the diagnosis of PE were $75 \%, 88 \%$, $88 \%$, and $74 \%$, respectively.

The results of diagnosis of PE by means of HCU are summarized in Table 3.

\section{Discussion}

The introduction of HCU imagers has been discussed controversially for image quality and decision-making in daily clinical practice, as earlier studies reported drawbacks of older portable ultrasound systems (19).

Recent studies $(15,19)$ have been using other HCU imagers, such as the Acuson P10 (Siemens Healthcare, Erlangen, Germany) or the OptiGo (Philips Medical Systems, Andover, CA, USA) as well as the Sono-Heart (SonoSite Inc., Bothell, WA, USA) and disclosed promising findings, but HCU imagers have not made their way into daily clinical workflow yet.

The present study was performed with a latest generation HCU device (VScan, GE Healthcare, Waukesha, WI, USA) with a plane 1.7-3.8 MHz transducer for two-dimensional imaging and a 3.5-inch display. The tested device proved good handling and image quality as well as quickness of use. It was evaluated before for its image quality in echocardiography by Prinz and Voigt (19) with satisfying results. But, to the best of our knowledge, it has not been investigated in the assessment of PE yet. 
Therefore, in the present study the yield of this new generation HCU imager for the diagnosis of PE was evaluated with contrast-enhanced MDCT as standard of reference.

Prevalence of PE was 56\% in our study population, which is comparable to the corresponding literature $(1,2)$. Sensitivity and specificity for the diagnosis of $\mathrm{PE}$ by means of HCU were $75 \%$ and $88 \%$, respectively, which is promising, in particular in consideration of the fact, that in all false-negative cases, the amount of PE was estimated to be $20 \mathrm{~mL}$ or less. As the pericardial cavity normally contains approximately $15-35 \mathrm{~mL}$ of pericardial fluid, these few amounts of PE of $<20 \mathrm{~mL}$ have to be considered to be either physiologically or at least hemodynamically irrelevant (20).

There are limitations of the present study. Only 36 patients were enrolled, and the examination with the HCU as well as the reading of the MDCT was carried out by only one operator, respectively. Naturally ultrasound is an operator-dependent modality and different operators might gain different results. Furthermore all patients were admitted to a cardiothoracic ICU with a self-evident high prevalence of PE.

In conclusion, the findings of this study indicate that modern HCU imagers provide a helpful approach for easy, reliable, and cost-effective detection of PE. Systematic use of HCU might improve surveillance of patients who are at risk of suffering from PE and it might help to diagnose PE before it becomes clinically relevant. Abandonment of radiation and quick bedside availability without the need of transportation are further advantages of patient surveillance by HCU systems.

\section{Conflict of interest: None.}

\section{REFERENCES}

1 Weitzman LB, Tinker WP, Kronzon I, et al. The incidence and natural history of pericardial effusion after cardiac surgery-an echocardiographic study. Circulation 1984;69:506-11

2 Stevenson LW, Child JS, Laks H, et al. Incidence and significance of early pericardial effusions after cardiac surgery. Am J Cardiol 1984;54:848-51

3 Nelson RM, Jenson CB, Smoot WM 3rd. Pericardial tamponade following open-heart surgery. J Thorac Cardiovasc Surg 1969;58:510-6
4 Hardesty RL, Thompson M, Lerberg DB, et al. Delayed postoperative cardiac tamponade: diagnosis and management. Ann Thorac Surg 1978;26:155-64

5 Fernando HA, Friedman HS, Lajam F, et al. Late cardiac tamponade following open-heart surgery: detection by echocardiography. Ann Thorac Surg 1977;24:174-7

6 Lee LN, Yang PC, Chang DB, et al. Ultrasound guided pericardial drainage and intrapericardial instillation of mitomycin $C$ for malignant pericardial effusion. Thorax 1994;49:594-5

7 Edler I, Gustafson A. Ultrasonic cardiogram in mitral stenosis; preliminary communication. Acta Med Scand 1957;159:85-90

8 Horowitz MS, Schultz CS, Stinson EB, et al. Sensitivity and specificity of echocardiographic diagnosis of pericardial effusion. Circulation 1974;50:239-47

9 Martin RP, Rakowski H, French J, et al. Localization of pericardial effusion with wide angle phased array echocardiography. Am J Cardiol 1978;42:904-12

10 Luo H, Chen M, Trento A, et al. Usefulness of a hand-carried cardiac ultrasound device for bedside examination of pericardial effusion in patients after cardiac surgery. Am J Cardiol 2004;94:406-7

11 Ligtvoet C, Rijsterborgh H, Kappen L, et al. Real time ultrasonic imaging with a hand-held scanner. Part I-technical description. Ultrasound Med Biol 1978;4:91-2

12 Roelandt J, Wladimiroff JW, Baars AM. Ultrasonic real time imaging with a hand-held-scanner. Part II-initial clinical experience. Ultrasound Med Biol 1978;4:93-7

13 Goodkin GM, Spevack DM, Tunick PA, et al. How useful is hand-carried bedside echocardiography in critically ill patients? J Am Coll Cardiol 2001;37:2019-22

14 Galderisi M, Santoro A, Versiero M, et al. Improved cardiovascular diagnostic accuracy by pocket size imaging device in non-cardiologic outpatients: the NaUSiCa (Naples Ultrasound Stethoscope in Cardiology) study. Cardiovasc Ultrasound 2010;8:51

15 Piccoli M, Trambaiolo P, Salustri A, et al. Bedside diagnosis and follow-up of patients with pleural effusion by a hand-carried ultrasound device early after cardiac surgery. Chest 2005;128:3413-20

16 Schleder S, Dendl LM, Ernstberger A, et al. Diagnostic value of a hand-carried ultrasound device for free intra-abdominal fluid and organ lacerations in major trauma patients. Emerg Med J 2012 Apr 19 [Epub ahead of print]

17 Schleder S, Dornia C, Poschenrieder F, et al. Bedside diagnosis of pleural effusion with a latest generation hand-carried ultrasound device in intensive care patients. Acta Radiol 2012;53:556-60

18 Goodman A, Perera P, Mailhot T, et al. The role of bedside ultrasound in the diagnosis of pericardial effusion and cardiac tamponade. J Emerg Trauma Shock 2012;5:72-5

19 Prinz C, Voigt JU. Diagnostic accuracy of a hand-held ultrasound scanner in routine patients referred for echocardiography. J Am Soc Echocardiogr 2011;24:111-6

20 Spodick DH. Pericardial diseases. In: Braunwald E, Zipes DP, Libby P, eds. Heart disease: a textbook of cardiovascular medicine. 6th edn. Philadelphia, PA: Saunders, 2001:1823-77 